\title{
Super- and multi-directive acoustic radiation by linear global modes of a turbulent jet
}

\author{
Oliver T. Schmidt, Aaron Towne, Tim Colonius \\ California Institute of Technology, Pasadena, CA, USA \\ Peter Jordan, Vincent Jaunet \\ Institut Pprime, CNRS - University of Poitiers - ENSMA, Poitiers, France \\ André V. G. Cavalieri \\ Instituto Tecnológico de Aeronáutica, São José dos Campos, Brazil \\ Guillaume A. Brès \\ CASCADE Technologies, Palo Alto, CA, USA
}

\begin{abstract}
The mean flow stability of a Mach 0.9 turbulent jet is investigated by means of global linear theory with a focus on acoustic effects. A novel class of resonant acoustic modes that are trapped within the potential core, and whose eigenvalues appear as discrete branches in the global stability spectrum, is studied in detail. A dispersion relation is reconstructed from the global modes, and shown to accurately predict energy bands observed in the PSD of a high-fidelity LES. Similarly, the acoustic far-field radiation patterns of the trapped modes are compared to the LES. A favorable agreement between the global mode waveforms and coherent structures educed from the LES is found for both the trapped acoustic wave component inside the core and the far-field radiation.
\end{abstract}

\section{Introduction}

Through a large body of numerical and experimental evidence, it is widely accepted that large scale coherent structures in turbulent jets can be described as instability waves, i.e., modal solutions to the governing equations. ${ }^{1}$ However, the role of such wave packets in jet noise generation remains disputable. As predicted by simplified models, ${ }^{2}$ super-directive acoustic radiation at low angles relative to the jet axis is commonly attributed to wave packets at low azimuthal wave numbers, whereas broadband noise is often interpreted as a result of fine-scale turbulence. ${ }^{3}$ In this paper, global modes of a high Reynolds number turbulent jet at Mach 0.9 are calculated as solutions to the eigenvalue problem obtained by linearizing the compressible Navier-Stokes around a turbulent mean flow under a normal waveform assumption. A carefully validated large eddy simulation (LES) of an isothermal subsonic turbulent jet issued from a convergent-straight nozzle serves as a reference, and provides the mean state. ${ }^{4}$ The LES accurately reproduces the configuration from a companion experiment. Figure 1 shows the instantaneous streamwise velocity perturbation from the first snapshot of the database. The jet shear-layer is initially turbulent, and the diameter-based Reynolds number is $R e \approx 10^{6}$. The reader is referred to Brès et al. ${ }^{4}$ for details.

Linearization about a turbulent mean flow has been invoked successfully in the past for jet configurations. For example, Gudmundsson \& Colonius ${ }^{5}$ and Cavalieri et al. ${ }^{6}$ space-marched the parabolized stability equations and showed that the axisymmetric and first helical modes of a turbulent jet correspond to linear wave packets in the potential core region. A first two-dimensional linear stability analysis of a turbulent mean flow obtained as a solution to Favre-averaged Navier-Stokes equations by Nichols and Lele ${ }^{7}$ demonstrated the feasibility of the global approach. The authors calculated global modes over a range of Mach numbers and jet heating ratios, and addressed the directivity of acoustic radiation emitted by global modes. The present paper is organized as follows. First, the numerical method is outlined in $\S I I$. In $\S I I I$, the linear

*oschmidt@caltech.edu 


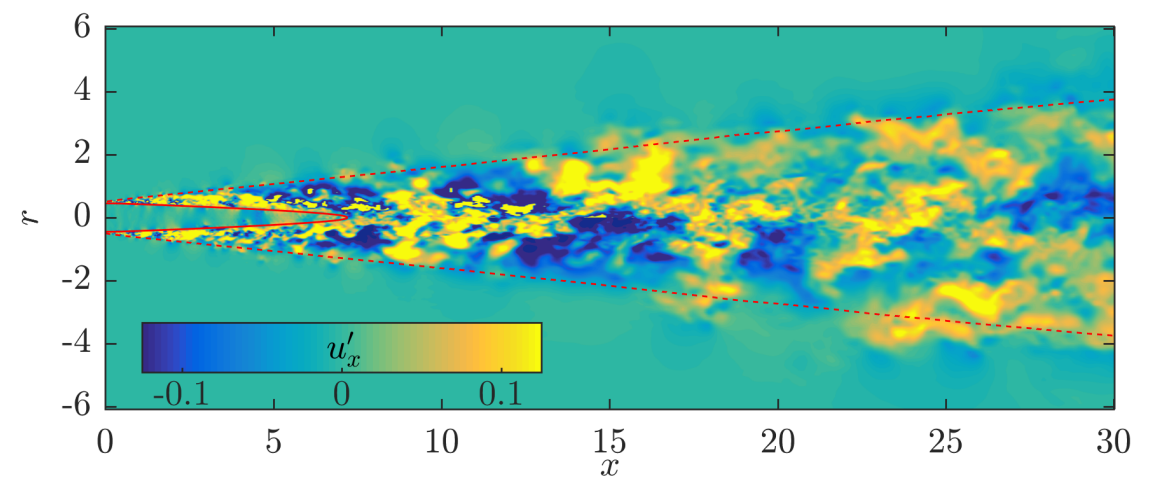

Figure 1. Instantaneous visualization of the streamwise perturbation velocity. The extent of the potential core is estimated as $\bar{u}=0.95$ (solid red line) and the jet cone as $\bar{u}=0.05$ (dashed red line).

dynamics within the potential core are examined. In particular, the occurrence of trapped acoustic modes recently observed and theoretically identified as eigensolutions of a cylindrical vortex sheet model by Towne et al. ${ }^{8}$, is examined. After establishing that the global ansatz accurately describes the latter phenomenon, the modal acoustic radiation to the far-field is addressed in $\S I V$. Finally, the findings and implications of our study are summarized in $\S \mathrm{V}$.

\section{Numerical method}

The global linear stability analysis is based on the linearized compressible Navier-Stokes equations

$$
\frac{\partial \mathbf{q}^{\prime}}{\partial t}=\mathcal{L} \mathbf{q}^{\prime}
$$

here represented by the linear operator $\mathcal{L}$ acting on the state vector $\mathbf{q}^{\prime}=\left\{\rho, u_{x}, u_{r}, u_{\theta}, T\right\}^{T}$ of the primitive variables density $\rho$, streamwise velocity $u_{x}$, radial velocity $u_{r}$, azimuthal velocity $u_{\theta}$, and temperature $T$. Upon insertion of the normal mode ansatz $\mathbf{q}^{\prime}(x, r, t)=\hat{\mathbf{q}}(x, r) \exp (\mathrm{i}(m \theta-\omega t))$ and spatial discretization, an eigenvalue problem of the form

$$
-\mathrm{i} \omega \hat{\mathbf{q}}=\mathbf{L} \hat{\mathbf{q}}
$$

is obtained, which can readily be solved by a standard shift-invert Arnoldi method for a given angular frequency $\omega$ and azimuthal wave number $m$. Fourth order summation by parts finite differences ${ }^{9}$ are used for the spatial discretization on a Cartesian grid. A computational domain $\Omega_{1}$ of size $x, r \in[0,25] \times[0,2.25]$ discretized on a $1150 \times 250$ grid point mesh is used for the near-field calculations presented in $\S I I I$, and an enlarged $x, r \in[0,30] \times[0,12]$ domain $\Omega_{2}$ with $950 \times 325$ grid points for the results presented in $\S \mathrm{IV}$. In both cases, a sufficiently large sponge region is used to prevent unwanted reflections from the boundaries.

Previous studies showed the the far-field noise of turbulent jets can be decomposed, almost entirely, into its leading three Fourier components. ${ }^{10,11}$ Our study is therefore restricted to the azimuthal wavenumbers $m=0,1,2$. Global stability spectra obtained for domain $\Omega_{1}$ and over a Strouhal number range $0.2 \leq S t=$ $\omega / 2 \pi \leq 1.9$ are shown in figure 2 . The two main features of the spectra are the continuous branch of KelvinHelmholtz (K-H) instability waves, and a number of discrete branches that emerge from the continuous spectrum within certain frequency bands. The discrete mode branches can be identified by their azimuthal and radial wavenumber pair $(m, n)$, as we will show in the following. It was confirmed through domain size and grid studies that the modes belonging to the latter branches are indeed discrete, whereas the K-H branches only get rendered discrete by the numerical discretization. The focus of this paper is on the discrete modes, which represent and acoustic phenomenon as we will demonstrate in the following.

\section{Trapped acoustic instability}

The occurrence of discrete modes is unexpected at first as high Reynolds number natural jets are known to be amplifier flows with no intrinsic instability mechanisms ${ }^{12}$. 

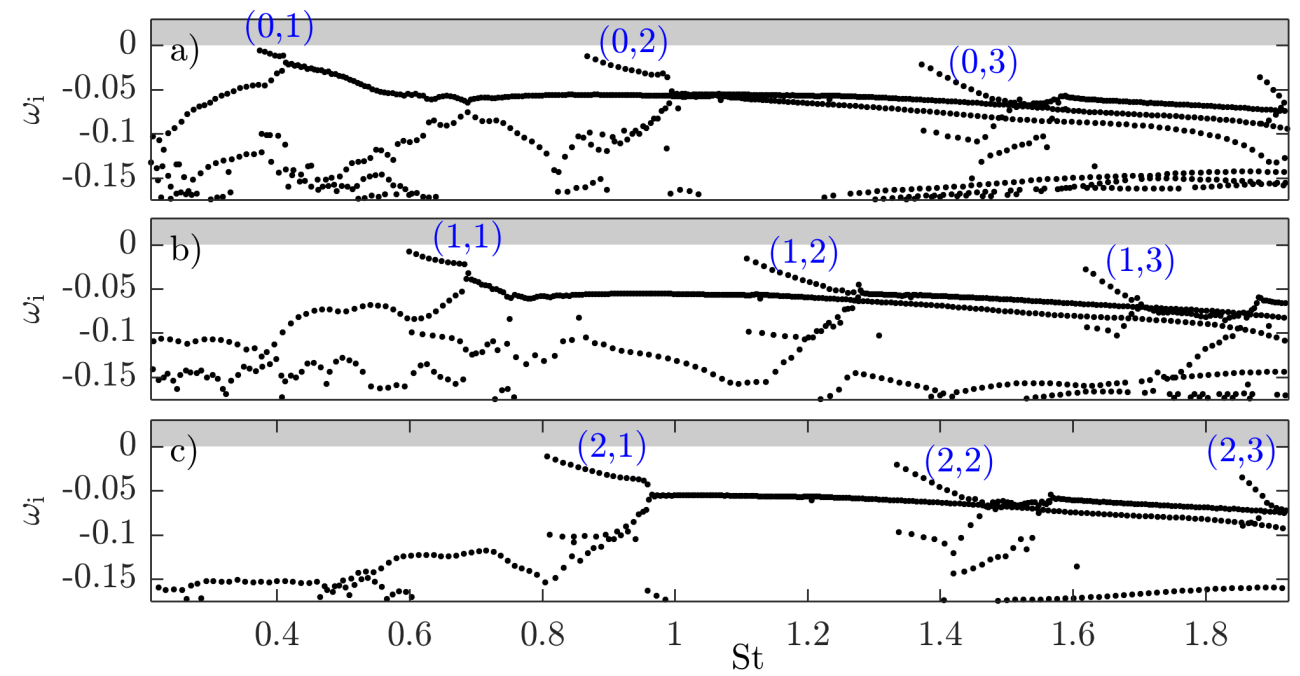

Figure 2. Global stability spectra: (a) $m=0$; (b) $m=1$; (c) $m=2$. Discrete mode branches are indicated by their azimuthal and radial wavenumber pair $(m, n)$ (blue).
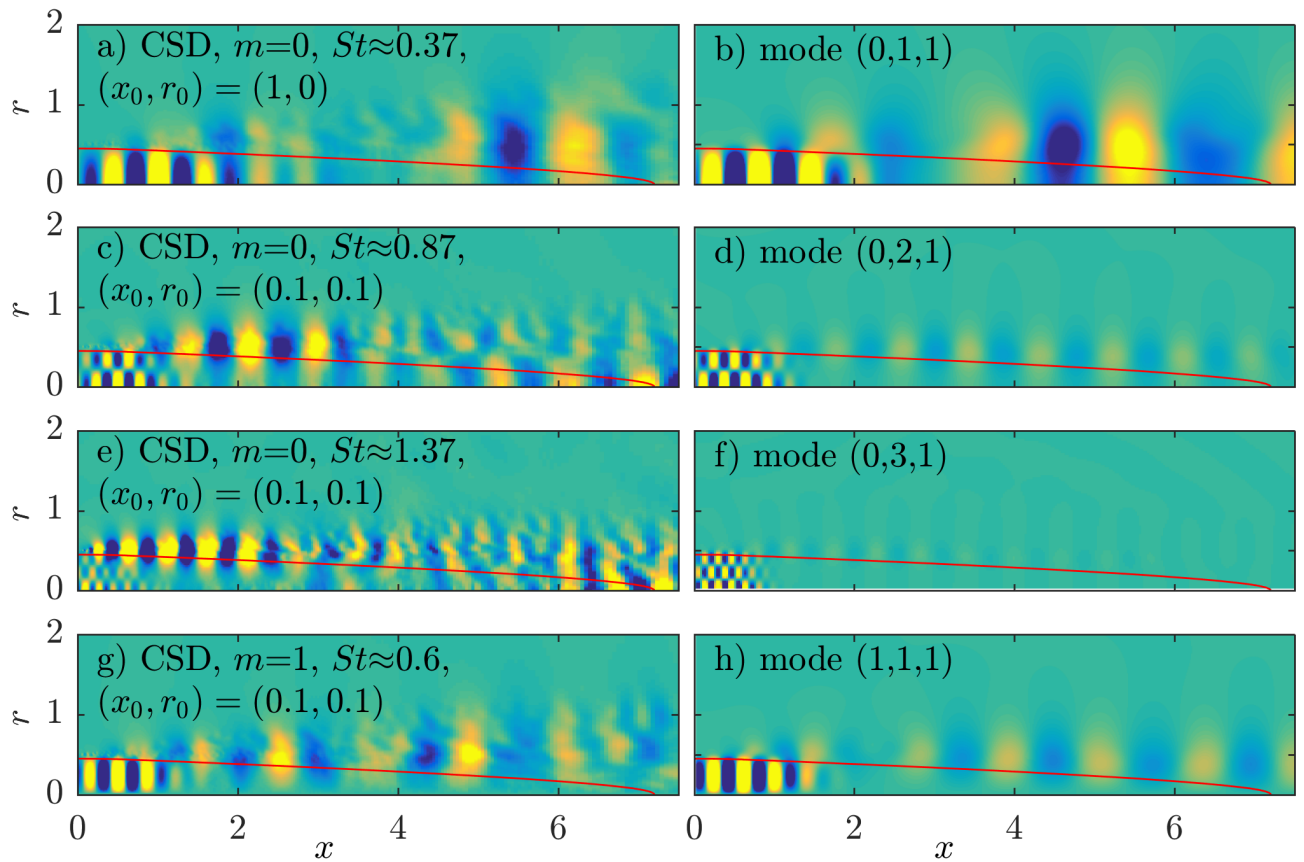

Figure 3. Comparison between LES cross-spectral destiny (left column) and discrete global modes (left column) at the corresponding azimuthal wavenumber and frequency. The CSD is computed with respect to the correlation location $\left(x_{0}, r_{0}\right)=(0.1,0.1)$ in order to isolate the acoustic instability in the potential core. The line of constant streamwise mean velocity $\bar{u}=0.95$ (solid red line) is indicated as in figure 1 .

Figure 3 compares different discrete global modes (left column) to the cross-spectral density (CSD) of the LES data at the corresponding modal frequency and azimuthal wavenumber. The CSD is obtained by crosscorrelating any point in the domain with the reference location $\left(x_{0}, r_{0}\right)=(0.1,0.1)$. This particular point close to the nozzle and the jet axis is chosen to extract the coherent pressure fluctuation in the potential core. The discrete modes are indicated by the triple $\left(m, n, n_{x}\right)$, where $n_{x}$ is the streamwise order to be discussed in the context of figure 4 below. Here, the leading mode $(m, n, 1)$ of each branch is chosen, i.e. the least damped mode, or equivalently, the mode with the lowest frequency. The radial order $n$ becomes apparent by comparing the modal waveforms near the nozzle. $n$ can readily be obtained as the number of anti-nodes in 
the radial direction. A striking resemblance between the CSD of the data and the global modes is observed in that region. The discrete global modes clearly represent an instability mechanism inside the potential core, which is likewise educed via the CSD from the data. The observed waveforms correspond to acoustic instabilities that experience the shear-layer as a pressure release surface, as shown by ${ }^{8}$.

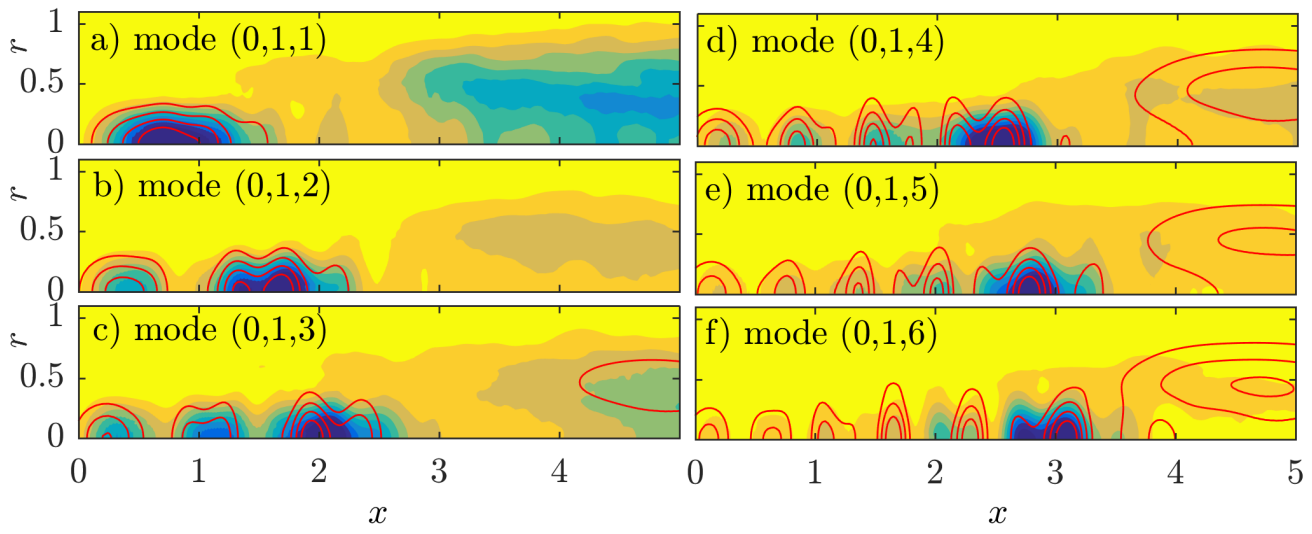

Figure 4. Comparison between LES pressure power spectral density (PSD, flooded contours, $\left.0 \leq \bar{P}_{p p} /\left\|\bar{P}_{p p}\right\|_{\infty} \leq 1\right)$ and global mode $\left(-, \hat{p}_{m} \hat{p}_{m}^{*}\right.$ at $20 \%, 40 \%, 60 \%$, and $80 \%$ of $\left.\max \left(\hat{p}_{m} \hat{p}_{m}^{*}\right)\right)$ for branch $(0,1)$.

The six modes comprising the $(0,1)$ branch are compared in figure 4 to the pressure PSD of the LES. The pressure PSD, here denoted as $\bar{P}_{p p}$, is estimated by means of Welch's method from 78 short-time (256 snapshots separated by $\Delta t=0.2$ acoustic time units) Fourier realizations with $50 \%$ overlap. Again, a favorable match between the linear modes and the educed structures is observed. We define the streamwise order, or wavenumber, $n_{x}$, as the number of anti-nodes in the streamwise direction. It can be seen that the streamwise extent of the trapped acoustic modes increases with increasing streamwise wavenumber. In our parallel investigation ${ }^{8}$, we show that this observation can be explained in terms of a frequency-dependent end condition which originates from the narrowing of the potential core.

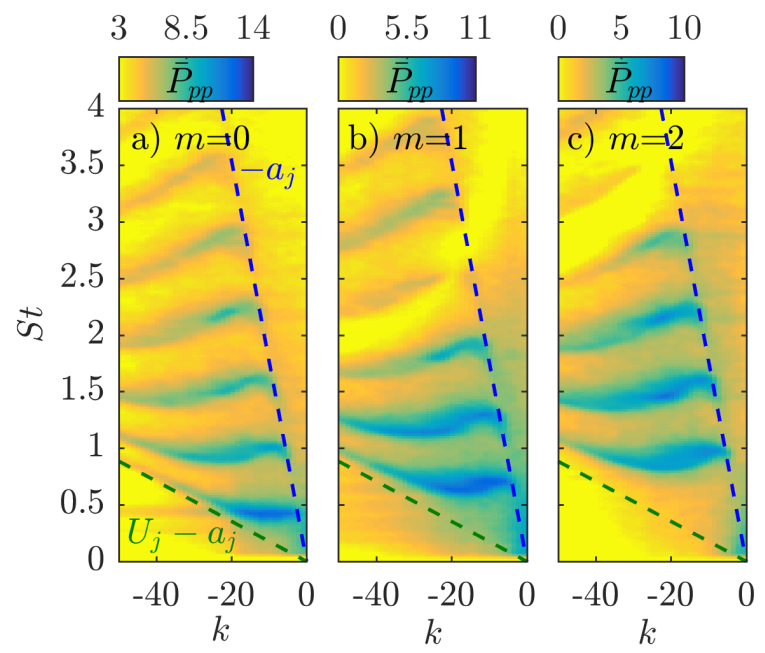

Figure 5. LES frequency-wavenumber diagram obtained from the pressure signal along the jet axis in the potential core $x \in[05]:$ (a) $m=0$; (b) $m=1$; (c) $m=2$.

The spectral energy content of the LES data in the potential core is examined in figure 5 in terms of frequency-wavenumber diagrams for the pressure PSD, where $k$ denotes the streamwise wavenumber. The diagrams are based on a Fourier decomposition of the pressure in the streamwise interval $x \in[05]$ at $r=0$ for $m=0$, and $r=0.1$ for $m>0$, respectively. By comparing the frequencies of the energy bands to the global mode spectra shown in figure 2, or alternatively by probing the data in terms of the CSD as shown in figure 3 , we readily identify the negative-wavenumber energy bands as the signatures of the trapped acoustic perturbations. The curved energy bands in the negative $k$-plane can be regarded as an empirical dispersion 
relation for the trapped acoustic instabilities. It can be seen that the trapped modes are confined within a segment that is limited by the negative jet speed of sound $-a_{j}$ and the difference between the jet velocity $U_{j}$ and $a_{j}$. Physically, this implies that the empirical dispersion relation describes the transition of acoustic duct modes that are confined within the potential core and propagate against the jet velocity, to acoustic free-stream modes that propagate in the ambient flow field ${ }^{8}$.

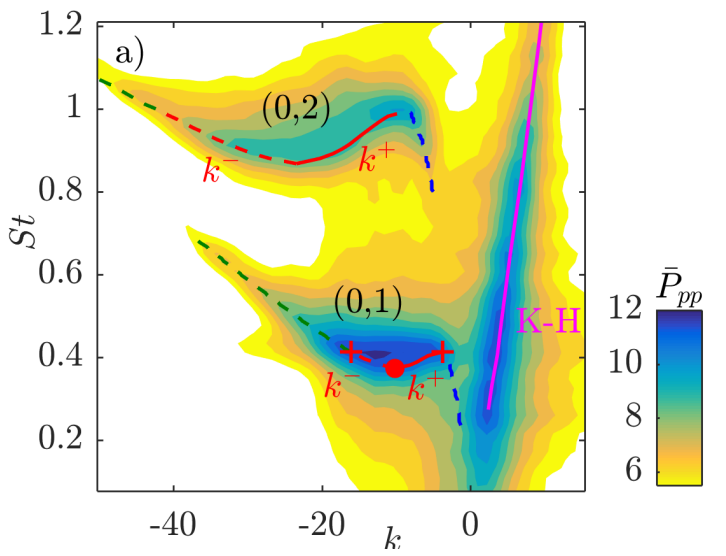

Figure 6. LES frequency-wavenumber diagram (contours, as in figure 5(a)) and reconstructed dispersion relations of the trapped acoustic modes and the $\mathrm{K}-\mathrm{H}$ instability for $\mathrm{m}=0$. The leading mode of the $(0,1)$ branch is marked as a red bullet symbol and the mode at the intersection of the $(0,1)$ branch and the continuous branch as a red ' + ' symbol.

Analogously, the spectral content of the global modes is extracted and the resulting reconstructed dispersion relations (lines) of the trapped modes and the K-H instability are compared to the LES frequencywavenumber diagram (contours) in figure 6 . The reconstructed dispersion relations are obtained by tracing the peaks in the wavenumber spectrum of the global modes. It can be seen that they closely follow the energy bands of the LES PSD. The green (acoustic duct-like behavior) and the blue segments (acoustic free-stream behavior) are constructed from the continuous modes to the left and to the right of the point where the discrete and the continuous branches intersect. The red line segments are associated with the discrete modes. Note that the local slope of the reconstructed dispersion relation determines the group velocity of the trapped acoustic modes. This means that the discrete modes possess two wave components, a $k^{-}$component of negative, and a $k^{+}$component of positive group velocity. The coexistence of the $k^{-}$ and the $k^{+}$waves explains the occurrence of the interference patterns seen in figure 4 in terms of a beating between the two components. The beating is facilitated by two end conditions that trap the acoustic mode in the streamwise direction. On the left, the nozzle impedance reflects the leftgoing wave. On the right, the narrowing of the potential core gives rise to frequency-dependent end condition. A similar phenomenon is known from waves in pipes of varying cross-section. The reader is referred to our parallel investigation ${ }^{8}$ for details on the resonant beating, and the implications of the two points of zero group velocity in the trapped acoustic wave dispersion relation.

\section{Acoustic far-field radiation}

Short-time Fourier realizations and leading proper orthogonal decomposition (POD) modes of the LES are compared in figure 7 for different frequencies and azimuthal wavenumbers. The POD is based on the 2-norm of the pressure in the far-field region (dashed red lines) in order to educe the dominant coherent contribution to the far-field sound. It can be seen that the POD identifies a super-directive acoustic beam which gets emitted from the shear-layer region around the end of the potential core in all cases. No such unified picture can be drawn from the short-time FFT modes. Different multi-directive or super-directive radiation patterns are observed. This observation can be attributed to the characteristic jitter ${ }^{13}$ of the jet. Note that the acoustic radiation patterns for frequencies that coincide with trapped acoustic mode frequencies, e.g., branch $(0,1)$ in $7(\mathrm{~d})$, do not qualitatively differ from the rest.

The computation of far-field acoustic radiation generated by linear global modes necessitates the use of an enlarged domain $\Omega_{2}$ as described in $\S I I$. The altered computational setup does not permit the computation of whole spectra due to a significant decrease of the convergence radius of the Arnoldi method due to spurious 

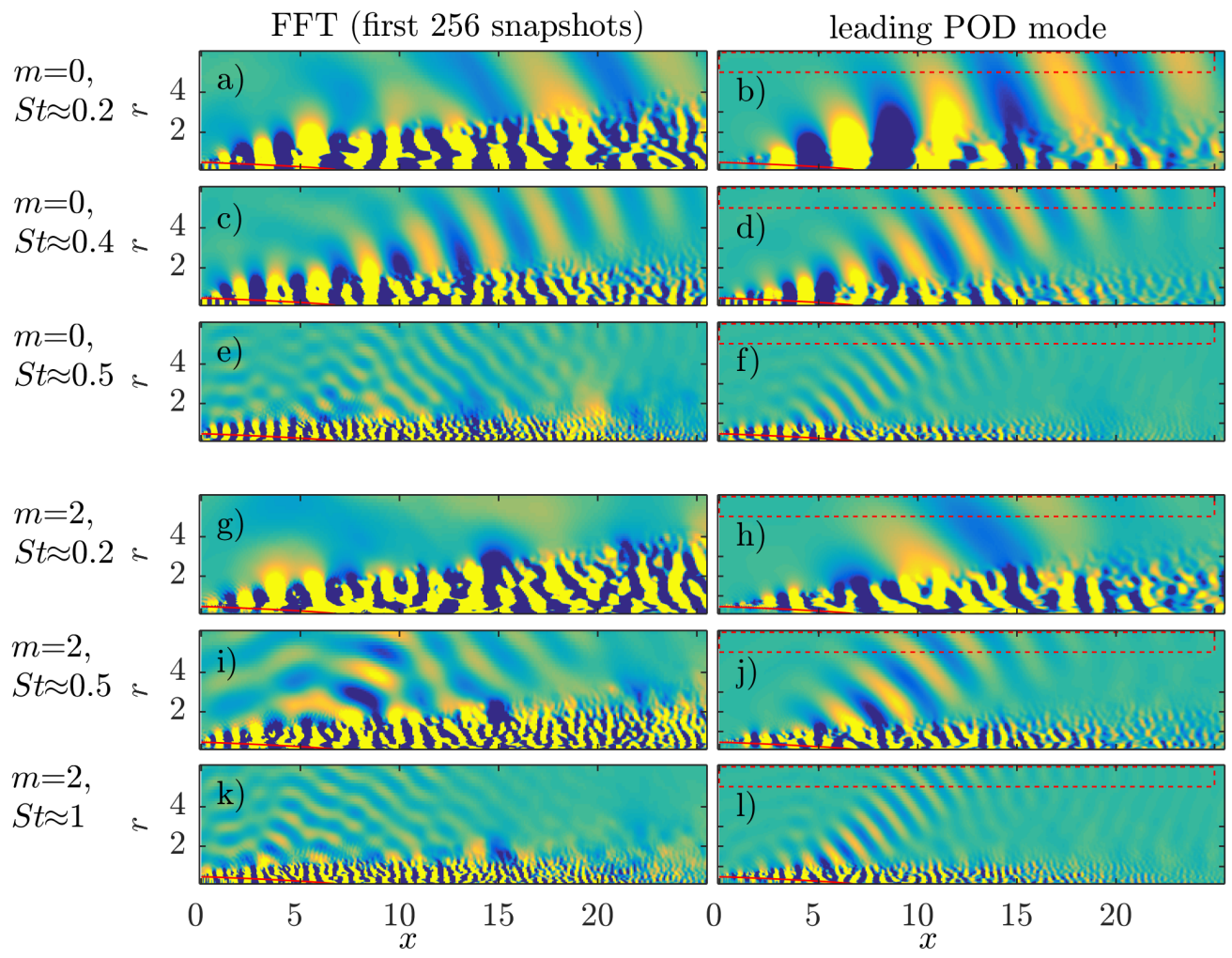

Figure 7. Comparison between LES short-time FFT (left column) and LES far-field weighted POD (right comlumn) for different azimuthal wavenumbers and frequencies.

modes. However, single global modes can readily be calculated by setting the shift parameter of the Arnoldi solver to the desired eigenvalue obtained on the $\Omega_{1}$ mesh.

In the following, we investigate the relevance of the trapped acoustic mechanism on the acoustic radiation. For that purpose, short-time Fourier realizations of the LES are compared to discrete global modes in figure 8. By first inspecting the global modes (right column), it can be seen that only mode $(0,1,1)$ has a clear super-directive radiation pattern whereas all other modes of higher radial or azimuthal wavenumber exhibit a multi-directive behavior. This indicates that the discrete modes are not directly related to the most energetic acoustic structures identified via POD in figure 7. However, an inspection of short-time Fourier modes of the LES reveals that the discrete global mode radiation patterns frequently reoccur in different statistically independent realizations of the FFT. Selected examples are presented in the left column of figure 8. It can be seen that the FFT realizations closely resemble the complex radiation patterns predicted by the global modes. However, only the super-directive radiation of the $(0,1,1)$ mode shown in figure $8(\mathrm{a}, \mathrm{b})$ also compares well with the most energetic POD mode. This suggests that even though the discrete mode radiation is clearly observed in the data, it does not play a dominant role for the overall jet noise. In the parallel experimental investigation, Jaunet et al. ${ }^{14}$ find coherence levels of about $10 \%$ between the trapped-mode branches $(0,1)$ and $(1,1)$, and the far-field at high polar angles. The far-field noise spectra of the LES shown in Brès et al. ${ }^{4}$, however, do not indicate the presence of the tapped acoustic instabilities, but are typically flat.

\section{Conclusion}

The linear global stability analysis was shown to accurately predict a novel class of trapped acoustic modes in the potential core of high subsonic turbulent jets. In the global stability spectra, the eigenvalues of these modes form branches of discrete modes which accurately predict the occurrence of energetic bands in the PSD of the LES in the frequency-wavenumber plane. Their modal shapes are found in good agreement with coherent structures educed from the LES via CSD. A dispersion relation of the trapped acoustic waves 

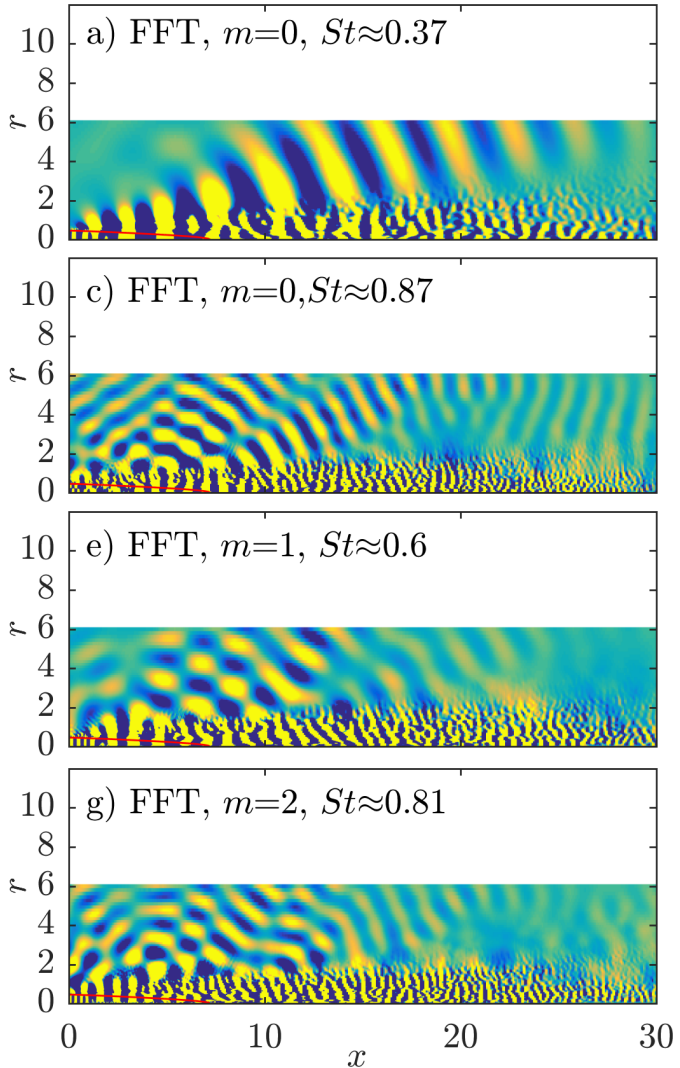
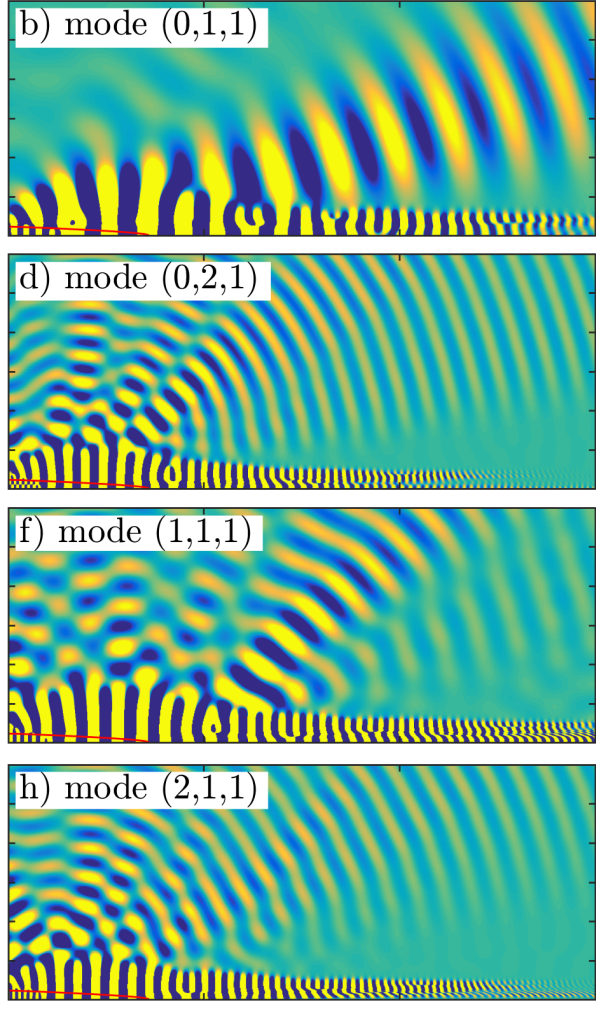

300
10
30

Figure 8. Comparison between selected short-time FFT realizations (left column) and global modes (right column) at the corresponding azimuthal wavenumbers and frequencies.

was constructed from the global modes, and shown to accurately follow the energy bands in the LES PSD. The relevance of the discrete modes in terms of jet noise was addressed by comparing their radiation patterns with short-time Fourier realizations of the LES. It was concluded that the discrete mode radiation patterns can be observed in the flow, but to not contribute to the overall jet noise in a prominent way. In a broader perspective, our findings demonstrate that acoustic effects in turbulent jets can be predicted by linear global theory to a large degree.

\section{Acknowledgments}

OTS gratefully acknowledges support by DFG grant no. 3114/1-1. AT and TC gratefully acknowledge support from the Office of Naval Research under contract N0014-11-1-0753. AVGC and PJ acknowledge support from the Science Without Borders program (project number A073/2013). The LES study was supported by NAVAIR SBIR project, under the supervision of Dr. John T. Spyropoulos. The main LES calculations were carried out on CRAY XE6 machines at DoD HPC facilities in ERDC DSRC.

\section{References}

${ }^{1}$ Jordan, P. and Colonius, T., "Wave packets and turbulent jet noise," Annual Review of Fluid Mechanics, Vol. 45, 2013, pp. 173-195.

${ }^{2}$ Crighton, D. G. and Huerre, P., "Shear-layer pressure fluctuations and superdirective acoustic sources," Journal of Fluid Mechanics, Vol. 220, 1990, pp. 355-368.

${ }^{3}$ Tam, C. K. W., Viswanathan, K., Ahuja, K. K., and Panda, J., "The sources of jet noise: experimental evidence," Journal of Fluid Mechanics, Vol. 615, 2008, pp. 253-292.

${ }^{4}$ Brès, G. A., Jaunet, V., Le Rallic, M., Jordan, P., Colonius, T., and Lele, S. K., "Large eddy simulation for jet noise: the importance of getting the boundary layer right," 21st AIAA/CEAS Aeroacoustics Conference, Vol. AIAA 2015-2535, Dallas, 
TX, 2015.

${ }^{5}$ Gudmundsson, K. and Colonius, T., "Instability wave models for the near-field fluctuations of turbulent jets," Journal of Fluid Mechanics, Vol. 689, 2011, pp. 97-128.

${ }^{6}$ Cavalieri, A. V. G., Rodríguez, D., Jordan, P., Colonius, T., and Gervais, Y., "Wavepackets in the velocity field of turbulent jets," Journal of Fluid Mechanics, Vol. 730, 2013, pp. 559-592.

${ }^{7}$ Nichols, J. and Lele, S., "Global mode analysis of turbulent high-speed jets," Center for Turbulence Research Annual Research Briefs, 2010.

${ }^{8}$ Towne, A., Cavalieri, A. V. G., Jordan, P., Colonius, T., Jaunet, V., Schmidt, O. T., and Brès, G. A., "Trapped acoustic waves in the potential core of subsonic jets," 22nd AIAA/CEAS Aeroacoustics Conference, 2016.

${ }^{9}$ Mattsson, K. and Nordström, J., "Summation by parts operators for finite difference approximations of second derivatives," Journal of Computational Physics, Vol. 199, No. 2, 2004, pp. 503-540.

${ }^{10}$ Michalke, A. and Fuchs, H. V., "On turbulence and noise of an axisymmetric shear flow," Journal of Fluid Mechanics, Vol. 70, No. 01, 1975, pp. 179-205.

${ }^{11}$ Cavalieri, A. V. G., Jordan, P., Colonius, T., and Gervais, Y., "Axisymmetric superdirectivity in subsonic jets," Journal of Fluid Mechanics, Vol. 704, 2012, pp. 388-420.

${ }^{12}$ Huerre, P. and Monkewitz, P. A., "Local and global instabilities in spatially developing flows," Annual review of fluid mechanics, Vol. 22, No. 1, 1990, pp. 473-537.

${ }^{13}$ Cavalieri, A. V. G., Jordan, P., Agarwal, A., and Gervais, Y., "Jittering wave-packet models for subsonic jet noise," Journal of Sound and Vibration, Vol. 330, No. 18, 2011, pp. 4474-4492.

${ }^{14}$ Jaunet, V., Jordan, P., Cavalieri, A. V. G., Towne, A., Colonius, T., Oliver, O. T., and Brès, G. A., "Tonal dynamics and sound in free and installed turbulent jets," 22nd AIAA/CEAS Aeroacoustics Conference, 2016. 